\title{
Resonant Transmission of Light Through Subwavelength Holes in Thick Metal Films
}

\author{
Jorge Bravo-Abad, Luis Martín-Moreno, and Francisco J. García-Vidal
}

(Invited Paper)

\begin{abstract}
In this paper, we study how extraordinary transmission through an array of holes in a metallic film appears as a function of the number of holes and their distribution. We have used a theoretical formalism able to analyze the optical properties of finite collections of apertures placed at arbitrary positions in a metallic film. First, we describe how the total transmission in a hexagonal two-dimensional hole array evolves as the number of holes in the array is increased. Second, we study the extraordinary transmission appearing in a single one-dimensional chain of holes. We find that the transmission through this structure is very sensitive to the incident polarization. Third, we show that a single hole flanked symmetrically by two finite chains of dimples can also present extraordinary transmission properties.
\end{abstract}

Index Terms-Extraordinary transmission, finite arrays, surface modes, surface plasmons.

\section{INTRODUCTION}

$\mathbf{T}$ HE first complete solution of the diffraction of electromagnetic (EM) radiation by a single hole in a metallic screen was published by Bethe in 1944 [1] and extended by Bouwkamp in 1954 [2]. This theory stated that, for an infinitesimally thin screen, if the radius of the hole $a$ is much smaller than the wavelength of the incident radiation $\lambda$, the normalizedto-area transmittance is proportional to $(a / \lambda)^{4}$. In other words, in the extreme subwavelength limit $(\lambda \gg a)$, only a negligible fraction of the electromagnetic energy flux that impinges on the hole is transmitted. More than 40 years later, Roberts showed that the effect of considering the finite thickness of the screen further decreases the transmission [3].

A real breakthrough in this field took place in 1998, when it was experimentally found that the transmission of light through an array of subwavelength holes in an optically thick metallic screen could be orders of magnitude greater than that expected from Bethe's theory [4].

The first experimental works [4]-[6] already suggested that the excitation of surface plasmon modes at the two interfaces of the system was the main mechanism responsible for the

Manuscript received February 28, 2006; revised July 4, 2006. This work was supported in part by the Spanish MCyT under Grant BES-2003-0374, Contract MAT2002-01534, and Contract MAT2002-00139, and in part by the European Commission (EC) under Project FP6-2002-IST-1-507879 and Project FP6-NMP4-CT-2003-505699.

J. Bravo-Abad and F. J. García-Vidal are with the Departamento de Física Teórica de la Materia Condensada, Universidad Autónoma de Madrid, Madrid, Spain.

L. Martín-Moreno is with the Departamento de Física de la Materia Condensada, ICMA-CSIC, Universidad de Zaragoza, Zaragoza, Spain.

Digital Object Identifier 10.1109/JSTQE.2006.881640 so-called extraordinary optical transmission. In order to clarify this point, different theoretical descriptions have been developed [7]-[11]. Currently, it is generally accepted that the extraordinary transmission phenomena correspond to different regimes of tunneling through surface electromagnetic modes located at the interfaces of the film [8], [12], [13]. Thus, extraordinary transmission through hole arrays is not restricted to the optical wavelengths but can be found in other frequency regimes [14]-[17] provided that the surface EM modes can be formed at the interfaces of the arrays. Therefore, from now on, we will refer to this phenomenon as extraordinary electromagnetic transmission (EET). Moreover, it has been recently demonstrated that extraordinary transmission also appears in photonic crystals in other structures [18]-[20] as well as in matter waves [21]. All these results suggest that the extraordinary transmission of waves assisted by the excitation of the surface modes is a very general phenomenon in wave physics.

Remarkably, in [8] it was found that EET is also obtained for perforated perfect conductors. This point was, very recently, theoretically explained in terms of the formation of surface EM modes in the corrugated surfaces of perfect conductors [22], [23].

In all these previous theoretical works, the hole arrays are treated as infinite periodic structures. However, the finite size of the sample must be taken into account in order to understand some of the fundamental features observed in the experiments [24]. A systematic study of the electromagnetic response of finite arrays of apertures in a metallic screen could also be useful to find new optical functionalities, as it was done before for the two-dimensional (2-D) analog of this system, i.e., in structures composed of 1-D apertures (slits and/or grooves) [25]-[31]. In addition, from an application point of view, the study of the EM near-fields corresponding to finite hole arrays seems to be a key issue for the development of the so-called plasmonic nanolithography [32].

On the other hand, very recently, the EET properties of finite arrays of holes have been studied experimentally in the terahertz regime [33]. The resonant transmission peak shown in these experiments is found at a frequency just above the cutoff frequency of the hole, which is different from that of the usual EET experiments, where the size of the holes are such that they are within the subwavelength regime. Up to now, and to the best of our knowledge, there has been no numerical confirmation of this finding.

In this paper, we study the electromagnetic properties of finite distributions of holes. In order to do this, we have used 


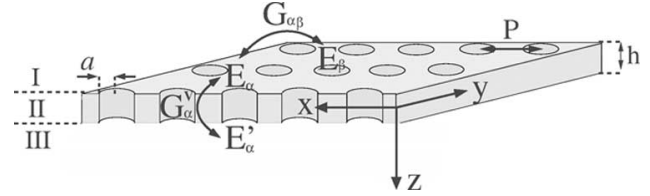

Fig. 1. Schematic picture of the structure under study and the corresponding reference system used. The physical meaning of each term of the system of linear equations (13) has also been added to this figure (see text for details on these magnitudes).

a formalism that is able to solve the electromagnetic response of a finite distribution (periodic or nonperiodic) of indentations in a metal film [34]. In this method, we apply perfect metal boundary conditions (PMBC) in the metallic regions. This will be a good approximation in both the microwave and terahertz regimes; therefore, quantitative agreement with experimental data is expected in these regimes. In the optical range, more realistic calculations can be done using this approach by enlarging the radii of the holes to take into account the increased cutoff found in real metals with respect to perfect metals [35], [36].

With the previous assumptions in mind, we will study the transmission properties of different finite distributions of holes. First, we will begin by analyzing the electromagnetic transmission properties of a hexagonal lattice of circular holes. We will present how the EET appears as the number of holes is increased. We will simulate, for the first time, the experiment made very recently by Miyamaru and Hangyo [33], confirming numerically the finite size effects found in that work.

Second, we will analyze the transmission properties of a single chain of holes, showing that this structure is very sensitive to the polarization of the incident radiation.

Finally, we show that it is possible to obtain extraordinary transmission through a single hole by flanking it with two finite chains of dimples located at the illuminated side of the film. As we will see, the total transmission is not affected by the corrugation in the nonilluminated side.

\section{THEORETICAL FORMALISM}

The type of structure analyzed in this paper and the reference system used are schematically shown in Fig. 1. Our aim is to study the electromagnetic response of a finite set of apertures distributed in a metallic screen. These apertures can be placed at arbitrary positions on the metallic film. A key point of our formalism is that we can consider different properties for each aperture, i.e., shape, dielectric constant and position of each aperture is defined separately from the rest.

Following Robert's work [3], through this paper, we will consider only the components of the electric field and the magnetic field that lie in the $x y$ plane $\left(\boldsymbol{E}_{t}\right.$ and $\boldsymbol{H}_{t}$, respectively). First of all, we assume a supercell of length $L_{x}$ and $L_{y}$ in the $x$ and $y$ directions, respectively, containing the structure we are interested in [Fig. 2(a)]. This supercell can be real, being the unit cell of an infinite periodic array [Fig. 2(b)], or fictitious, corresponding to a structure with a finite number of indentations given by $N$. In this last case, at the end of the procedure, the limit $L_{x}, L_{y} \rightarrow \infty$ must be taken [Fig. 2(c)].

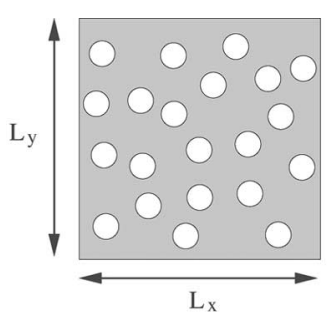

(a)

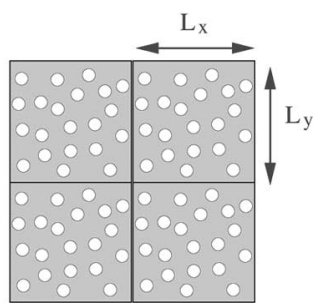

(b)

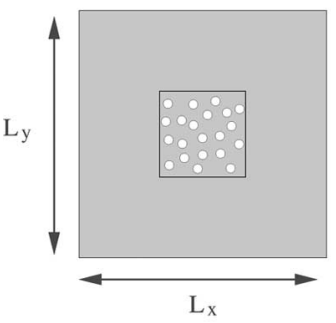

(c)

Fig. 2. Diagrams of the supercell used in the formalism described in the text.

Second, we divide the space within the supercell in three regions (labeled as I to III in Fig. 1). The width of the film is given by $h$. We assume an external plane wave impinging with a wavevector $\boldsymbol{k}_{0}$ from the top and whose direction is defined by a polar angle $\left(\theta_{\text {inc }}\right)$ and an azimuthal angle $\left(\phi_{\text {inc }}\right)$.

If we take the origin of the $z$-axis in the middle of the film, the fields $\left|\boldsymbol{E}_{t}\right\rangle$ and $\left|\boldsymbol{H}_{t}\right\rangle$ can be written as follows.

In Region I, the transversal fields admit the expansions

$$
\begin{gathered}
\left|\boldsymbol{E}_{t}\right\rangle=\delta_{\boldsymbol{k} \sigma, \boldsymbol{k}_{0} \sigma_{0}}\left|\vec{\phi}_{\boldsymbol{k} \sigma}^{\mathrm{I}+}\right\rangle+\sum_{\boldsymbol{k} \sigma} \rho_{\boldsymbol{k} \sigma}\left|\vec{\phi}_{\boldsymbol{k} \sigma}^{\mathrm{I}}\right\rangle \\
-\boldsymbol{u}_{z} \times\left|\boldsymbol{H}_{t}\right\rangle=Y_{\boldsymbol{k} \sigma}^{\mathrm{I}} \delta_{\boldsymbol{k} \sigma, \boldsymbol{k}_{0} \sigma_{0}}\left|\vec{\phi}_{\boldsymbol{k} \sigma}^{\mathrm{I}+}\right\rangle-\sum_{\boldsymbol{k} \sigma} Y_{\boldsymbol{k} \sigma}^{\mathrm{I}} \rho_{\boldsymbol{k} \sigma}\left|\vec{\phi}_{\boldsymbol{k} \sigma}^{\mathrm{I}}\right\rangle
\end{gathered}
$$

where wave vector $\boldsymbol{k}$ is defined as $\boldsymbol{k} \equiv\left(k_{x}, k_{y}\right)$ and the subscript $\sigma$ defines the polarization, being $\sigma=1$ for s-polarization and $\sigma=2$ for p-polarization. Function $Y_{k \sigma}^{\mathrm{I}}$ corresponds to the impedance of region I, i.e., $Y_{k 1}^{\mathrm{I}}=k_{z} / k_{0}$ and $Y_{k 2}^{\mathrm{I}}=k_{0} / k_{z}$, being $k_{z}=\sqrt{k_{0}^{2}-k^{2}}$ with $k=|\boldsymbol{k}|$. Coefficients $\rho_{\boldsymbol{k} \sigma}$ are the reflection amplitudes. In real space, the plane waves $\left|\vec{\phi}_{\boldsymbol{k} \sigma}^{ \pm \pm}\right\rangle$are

$$
\left\langle\boldsymbol{r} \mid \vec{\phi}_{\boldsymbol{k} \sigma}^{I \pm}\right\rangle=\exp \left[ \pm i k_{z}(z+h / 2)\right] \vec{\psi}_{\boldsymbol{k} \sigma}(x, y)
$$

with

$$
\vec{\psi}_{\boldsymbol{k} \sigma}(x, y)=\frac{\exp \left[i\left(k_{x} x+k_{y} y\right)\right]}{\sqrt{L_{x} L_{y}}}\left\{\begin{array}{ll}
\left(\begin{array}{c}
-k_{y} / k \\
k_{x} / k
\end{array}\right), & \text { if } \sigma=1 \\
\left(\begin{array}{c}
k_{x} / k \\
k_{y} / k
\end{array}\right), & \text { if } \sigma=2
\end{array} .\right.
$$


Notice that the bivectors are normalized to obtain $\left\langle\vec{\phi}_{\boldsymbol{k} \sigma}^{I \pm} \mid \vec{\phi}_{\boldsymbol{k} \sigma}^{\mathrm{I}}\right\rangle=1$.

In Region II, for $-h / 2<z<h / 2$ we can write

$$
\begin{aligned}
\left|\boldsymbol{E}_{t}\right\rangle & =\sum_{\alpha}\left[A_{\alpha}\left|\vec{\chi}_{\alpha}^{\mathrm{II}+}\right\rangle+B_{\alpha}\left|\vec{\chi}_{\alpha}^{\mathrm{II}-}\right\rangle\right] \\
-\boldsymbol{u}_{z} \times\left|\boldsymbol{H}_{t}\right\rangle & =\sum_{\alpha} Y_{\alpha}^{\mathrm{II}}\left[A_{\alpha}\left|\vec{\chi}_{\alpha}^{\mathrm{II}+}\right\rangle-B_{\alpha}\left|\vec{\chi}_{\alpha}^{\mathrm{II}-}\right\rangle\right]
\end{aligned}
$$

where $\alpha$ is an index that labels each eigenmode considered in this problem. In other words, each $\alpha$ defines a certain waveguide mode inside a certain aperture. The propagation constant along the $z$ direction of eigenmode $\alpha$ is given by $q_{\alpha z}$. Notice that this compact notation allows us to develop a very general formalism valid for any shape and location of the apertures.

In (6), $Y_{\alpha}^{\mathrm{II}}$ defines the impedance of the corresponding mode and is given by $Y_{\alpha}^{\mathrm{II}}=q_{\alpha z} / k_{0}$ in the case of TE modes, while $Y_{\alpha}^{\mathrm{II}}=k_{0} / q_{\alpha z}$ for TM modes.

The eigenmode is given by $\left|\vec{\chi}_{\alpha}^{\mathrm{II} \pm}\right\rangle$, which can be written in real space as

$$
\left\langle\boldsymbol{r} \mid \vec{\chi}_{\alpha}^{\mathrm{II} \pm}\right\rangle=\exp \left( \pm i q_{\alpha} z\right) \vec{\varphi}_{\alpha}\left(x-x_{\alpha}, y-y_{\alpha}\right) .
$$

Functions $\vec{\varphi}_{\alpha}(x, y)$ have analytical expressions for circular and rectangular holes [37]. In order to consider shapes different from these simple ones, the corresponding eigenmodes could be calculated using numerical methods such as the transfermatrix [38] or the multiple multipole method [39].

Finally, in region III, the EM fields can be also expanded in terms of plane waves as

$$
\begin{aligned}
\left|\boldsymbol{E}_{t}\right\rangle & =\sum_{\boldsymbol{k} \sigma} \tau_{\boldsymbol{k} \sigma}\left|\vec{\phi}_{\boldsymbol{k} \sigma}^{\mathrm{III}+}\right\rangle \\
-\boldsymbol{u}_{z} \times\left|\boldsymbol{H}_{t}\right\rangle & =\sum_{\boldsymbol{k} \sigma} Y_{\boldsymbol{k} \sigma}^{\mathrm{III}} \tau_{\boldsymbol{k} \sigma}\left|\vec{\phi}_{\boldsymbol{k} \sigma}^{\mathrm{III}+}\right\rangle
\end{aligned}
$$

where $Y_{\boldsymbol{k} \sigma}^{\mathrm{III}}$ is the impedance corresponding to region III and $\tau_{\boldsymbol{k} \sigma}$ are the transmission amplitudes. In region III we have defined the plane waves as

$$
\left\langle\boldsymbol{r} \mid \vec{\phi}_{\boldsymbol{k} \sigma}^{\mathrm{III} \pm}\right\rangle=\exp \left[ \pm i k_{z}(z-h / 2)\right] \vec{\psi}_{\boldsymbol{k} \sigma}(x, y) .
$$

In the next step, we apply the correspoding matching conditions on $\left|\boldsymbol{E}_{t}\right\rangle$ and $\left|\boldsymbol{H}_{t}\right\rangle$ at the interfaces of the system and we project them over the plane waves and the eigenmodes of the apertures, respectively. Once the set $\left\{A_{\alpha}, B_{\alpha}\right\}$ is calculated, we can compute all the electromagnetic magnitudes for this problem and, in particular, the transmission properties.

Now, we will see how to establish a connection between a solution based on a modal expansion of the fields and a formalism based on a 3-D Green's tensor. First, we introduce a new set of variables $\left\{E_{\alpha}, E_{\alpha}^{\prime}\right\}$ defined by

$$
\begin{aligned}
& E_{\alpha}=A_{\alpha}+B_{\alpha} \\
& E_{\alpha}^{\prime}=-\left(A_{\alpha} e_{\alpha}+B_{\alpha} e_{\alpha}^{-1}\right)
\end{aligned}
$$

where $e_{\alpha}=\exp \left(\imath q_{\alpha z} h\right)$.

Using these definitions in the projected matching equations we obtain the following new set of linear equations for the unknowns $\left[E_{\alpha}, E_{\alpha}^{\prime}\right]$

$$
\begin{aligned}
& \left(G_{\alpha \alpha}^{\mathrm{I}}-\epsilon_{\alpha}\right) E_{\alpha}+\sum_{\beta \neq \alpha} G_{\alpha \beta}^{\mathrm{I}} E_{\beta}-G_{\alpha}^{\mathrm{V}} E_{\alpha}^{\prime}=I_{\alpha}^{\mathrm{ext}} \\
& \left(G_{\gamma \gamma}^{\mathrm{III}}-\epsilon_{\gamma}\right) E_{\gamma}^{\prime}+\sum_{\nu \neq \gamma} G_{\gamma \nu}^{\mathrm{III}} E_{\nu}^{\prime}-G_{\gamma}^{\mathrm{V}} E_{\gamma}=0
\end{aligned}
$$

where we have defined

$$
\begin{aligned}
G_{\alpha \beta}^{\mathrm{I}, \mathrm{III}} & =\imath \sum_{\boldsymbol{k} \sigma} Y_{\boldsymbol{k} \sigma}^{\mathrm{I}, \mathrm{III}} I_{\boldsymbol{k} \sigma, \alpha}^{*} I_{\boldsymbol{k} \sigma, \beta} \\
I_{\alpha}^{\mathrm{ext}} & =2 \imath Y_{\boldsymbol{k}_{0} \sigma_{0}}^{\mathrm{I}} I_{\boldsymbol{k}_{0} \sigma_{0}, \alpha}^{*} \\
\epsilon_{\alpha} & =-\imath Y_{\alpha}^{\mathrm{II}}\left(1+\Phi_{\alpha}\right) /\left(1-\Phi_{\alpha}\right) \\
G_{\alpha}^{V} & =-2 \imath Y_{\alpha}^{\mathrm{II}} \sqrt{\Phi_{\alpha}} /\left(1-\Phi_{\alpha}\right)
\end{aligned}
$$

where $I_{\boldsymbol{k} \sigma, \alpha}=\exp \left[i\left(k_{x} x_{\alpha}+k_{y} y_{\alpha}\right)\right]\left\langle\vec{\psi}_{\boldsymbol{k} \sigma}^{\mathrm{I}} \mid \vec{\varphi}_{\alpha}\right\rangle \quad$ and $\quad \Phi_{\alpha}=$ $\exp \left(2 \imath q_{\alpha z} h\right)$.

The physical picture that emerges from the linear system of equations (13) is sketched in Fig. 1. The mode $\alpha$ is characterized by two field amplitudes, $E_{\alpha}$ and $E_{\alpha}^{\prime}$, the former corresponding to the illuminated side while the latter to the exit side.

The electromagnetic coupling between the objects $\alpha$ and $\beta$ through radiative modes is given by $G_{\alpha \beta}$, both in the input and the ouput surface. This coupling gives the terms $\sum_{\beta} G_{\alpha \beta}^{\mathrm{I}} E_{\beta}$ and $\sum_{\nu} G_{\gamma \nu}^{\mathrm{III}} E_{\nu}^{\prime}$ in the system of equations (13).

The coupling between the top and the bottom interfaces is controlled by $G_{\alpha}^{\mathrm{V}}$, this quantity gives the terms $G_{\alpha}^{\mathrm{V}} E_{\alpha}$ and $G_{\gamma}^{\mathrm{V}} E_{\gamma}^{\prime}$. In addition, we have to take into account the external illumination over the holes. In our formalism this is described by $I_{\alpha}^{\text {ext }}$, which, as it is expected, only appears in the matching equation corresponding to the input side and is given basically by the overlap between the incident beam and the eigenmode $\alpha$ [see (15)]. Spatially localized incident beams can also be treated within this technique. We could include them merely by introducing their projections over the corresponding waveguide eigenmodes. Finally, we can understand the magnitude $\epsilon_{\alpha}$ as coming from the back and forth motion of the photon inside the aperture $\alpha$.

Therefore, we have reduced our problem to solving the set of modal amplitudes $\left\{E_{\alpha}, E_{\alpha}^{\prime}\right\}$ at the apertures of the metallic film. In other words, in our formalism, we have to consider only the EM fields at the two surfaces of the openings. This increases to a great extent the efficiency of the proposed approach compared to the numerical methods that have to treat the entire metal surface numerically.

Importantly, the linear system of equations (13) hold for both an infinite periodic and a finite array of holes; the difference between these two cases lies in the calculation of $G_{\alpha \beta}$. In the case of infinite periodic structure, and using the corresponding overlapping integrals, the computation of $G_{\alpha \beta}$ is straightforward using (14). However, for finite systems the limit $L_{x}, L_{y} \rightarrow \infty$ must be taken. Then, instead of the previous discrete sum we have an integral that can be written as $G_{\alpha \beta}=\langle\alpha|\hat{G}| \beta\rangle$, where the dyadic $\hat{G}\left(\vec{r}_{\|}, \vec{r}_{\|}^{\prime}\right)=\left\langle\vec{r}_{\|}|\hat{G}| \vec{r}_{\|}^{\prime}\right\rangle$ [with $\left.\vec{r}_{\|}=(x, y)\right]$ can obtained 
from

$$
\hat{G}_{i j}\left(\vec{r}_{\|}, \vec{r}_{\|}^{\prime}\right)=g(d) \delta_{i j}+\left(2 \delta_{i j}-1\right) \frac{\partial^{2} g(d)}{\partial d_{i} \partial d_{j}}
$$

where $i$ and $j$ label either $x$ or $y, \delta_{i j}$ is Kronecker's delta, $\vec{d} \equiv$ $k_{0}\left(\vec{r}_{\|}-\vec{r}_{\|}^{\prime}\right)$ and $g(d)=k_{0} \exp (\imath d) /(2 \pi d)$.

Now, let us focus on the expression of the transmittance within the present formalism. We can write the total transmitted power across the holes as

$$
W=\Re\left\{\int d \boldsymbol{r} \boldsymbol{E}_{t}(\boldsymbol{r})\left[-\boldsymbol{u}_{z} \times \boldsymbol{H}^{*}(\boldsymbol{r})\right]\right\} .
$$

Using the expansions for $\boldsymbol{E}_{t}(\boldsymbol{r})$ and $\boldsymbol{H}_{t}(\boldsymbol{r})$ for the transmission region given in (8) and (9) and taking into account the expression for $\tau_{\boldsymbol{k} \sigma}$ given in terms of the coefficients $\left\{E_{\alpha}, E_{\alpha}^{\prime}\right\}$, we obtain

$$
W=\Re\left\{\sum_{\alpha, \beta} G_{\alpha \beta} E_{\alpha}^{\prime} E_{\beta}^{\prime *}\right\} .
$$

Similarly, we could compute the total transmitted power using the EM fields inside the holes, i.e., using the expansion in modal coefficients given in (5) and (6)

$$
W=\Re\left\{\sum_{\alpha} G_{\alpha}^{V} E_{\alpha} E_{\alpha}^{\prime *}\right\} .
$$

If we take into account that the total incident power incident on the structure is $W_{0}=Y_{\boldsymbol{k}_{0} \sigma_{0}}$, then the total transmittance $t$ can be computed as $t=W / W_{0}$.

In all the calculations shown in this paper, we have assumed that the metal is a perfect conductor. Therefore, all the results presented here are scalable and we have chosen the period $P$ of the arrays as a length unit.

\section{EVOLUTION OF EET AS THE NUMBER OF HOLES IS INCREASED}

In this section, we present how the transmission evolves from the case of a single hole, where the transmittance decreases monotonically with wavelength, to the case of an infinite periodic array of holes, where resonant transmittance peak(s) appear in the spectrum. We will begin by considering that the holes are disposed forming a 2-D hexagonal lattice. We have chosen this structure in order to simulate the experimental findings reported very recently in the terahertz regime [33]. Normal incidence and $p$-polarization for the incident plane wave is assumed in the following calculations. In addition, we consider that only the two least evanescent modes are included in the calculation. We have checked that this assumption gives accurate numerical results in the wavelength interval considered through this paper.

Fig. 3 shows the total transmission as a function of the number of holes $N$ for finite arrays whose size ranges from 10 to 105. The transmission is normalized to the area of the holes and divided by the corresponding $N$. This will be the normalization used in all the transmission spectra presented in this paper. The thickness of the metal film in both the panels is $h / P=0.44$ (corresponding to the metal thickness of the samples analyzed in experiment [33]) and two different radii of the holes are studied:

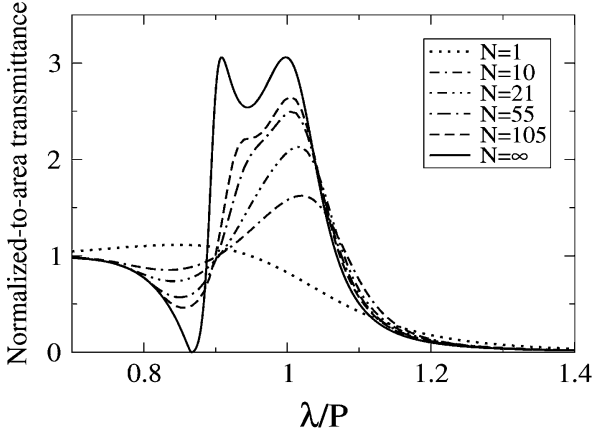

(a)

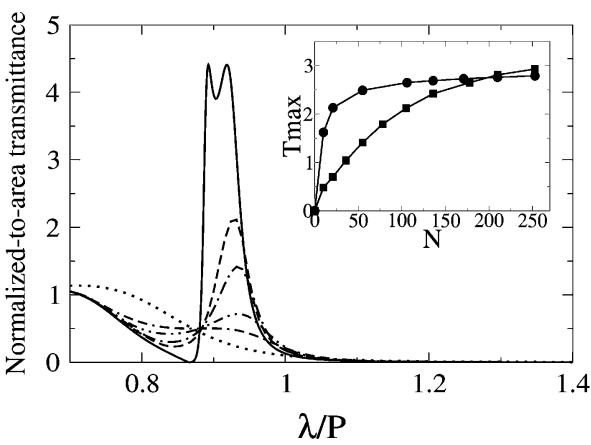

(b)

Fig. 3. (a) Normalized-to-area total transmittance of a hexagonal array of circular holes as a function of the wavelength for several values of the number of holes $N$. The geometrical parameters defining the structure are $a / P=0.3$ and $h / P=0.44$. Normal incidence and p-polarization is assumed for the incident plane wave. (b) Same as (a) but in this case for $a / P=0.25$. Inbox corresponds to the dependence of the maximum transmittance $\left(T_{\max }\right)$ as a function of $N$ for $a / P=0.3$ (circles) and $a / P=0.25$ (squares).

(a) $a / P=0.3$ (as in [33]) and (b) $a / P=0.25$. The resulting transmission spectra for both a single hole and an infinite periodic array of holes are also shown in these panels (see solid and dotted lines, respectively). For an infinite array, there are two main features appearing in the spectra. First, a well-defined minimum (known as Wood's anomaly) located at a wavelength $\left(\lambda_{\mathrm{W}}\right)$ in which a diffracted mode changes its character from propagating to evanescent. In the case of a hexagonal lattice, the largest $\lambda$ that satisfies this condition is $\lambda_{W}=\sqrt{3} / 2 P$. Second, two transmission peaks emerge at wavelengths slightly larger than $\lambda_{\mathrm{W}}$. These two peaks are the fingerprints of the EET phenomenon and can be explained in terms of the formation of surface EM modes at each metal-vacuum interface that are electromagnetically coupled via the holes [8].

Now we discuss the effect that the finite size of the array has on the transmittance spectrum. It is worth noting that we can define a resonant interval $(\lambda=0.75-1.25 P$ for the case $a / P=0.3$ and $\lambda=0.75-1 P$ for $a / P=0.25)$ in which a large number of holes are necessary to reproduce the main features found in the infinite periodic system. Outside this wavelength interval, the transmittance spectrum is independent on the number of holes. Importantly, as can be seen in the inbox of Fig. 3(b), the maximum of transmittance found in this interval $\left(T_{\max }\right)$ tends asymptotically to the value corresponding to an infinite array. It is possible to define a critical size $N_{c}$ for which we can state that 
the main properties of an infinite hole array has been reached. Fig. 3 clearly demonstrates that $N_{c}$ is completely governed by the ratio $a / P, N_{c}$ increases dramatically as $a / P$ decreases.

Finally, we will end this section with a comment on the experiment of Miyamaru and Hangyo [33]. In this experiment, they analyze hole arrays in which $N$ ranges from 1 to 21 . They found a linear dependence of the transmittance at the peak frequency on the number of holes. We also obtain the same linear dependence for the same sizes of the array [see the circles in the inbox of Fig. 3(b)]. However, we have found that this linear dependence is only valid for small values of $N$ as for $N>20$ the dependence of $T_{\max }$ as a function of $N$ takes the form $T_{\infty}(1-1 / N)$, something that could not be experimentally observed in [33] due to their maximum array size.

\section{Single Chain OF Holes: Minimal System SHOWING EET}

In this section, we will present a chain of holes as the simplest structure showing EET properties. We will use the same geometrical parameters as those used in the last section $(a / P=0.25$ and $h / P=0.44)$ in order to show that the numerical results shown here could be straightforward measured in the terahertz regime by using a similar experimental setup as that described in [33]. Notice that, as we have mentioned in Section I, we expect a quantitative agreement between the theory and the experimental data for that case.

Fig. 4(a) shows the evolution of the transmittance as a function of the number of holes in the linear chain $(N)$. We have assumed that the chain is illuminated by a plane wave that impinges normally with the electric field pointing along the direction of the chain. As can be observed in this figure, as we increase $N$, a transmittance peak emerges at $\lambda \approx P$. This shows that EET is already present in this 1-D arrangement of holes.

In order to study how the resonant peak appears in this structure, we have plotted the dependence of the maximum value of the transmission $\left(T_{\max }\right)$ as a function of the number of holes. The result is rendered in the inbox of Fig. 4(a). As can be seen, for small $N, T_{\max }$ increases linearly with $N$, while for enough large $N$ the transmittance peak approaches asymptotically (following again a $1 / N$ dependence) the value corresponding to an infinite chain of holes. In the same way as we did in the previous section, we can define a critical number of holes $N_{c}$ that determines the point at which the structure almost reaches the transmission properties of its corresponding infinite counterpart. For the geometrical parameters we are considering in this case, $N_{c} \approx 200$.

It is also interesting to compare the maximum value of transmittance obtained for an infinite chain of holes $\left(T_{\max }^{1-\mathrm{D}}=3.35\right)$ with that corresponding to the case of an infinite periodic 2-D square array $T_{\max }^{2-\mathrm{D}}=5.09$ ) for the same geometrical parameters. Notice that the transmittance at the resonant peak is increased by about $34 \%$.

It is worth commenting that in the case of a incident electric field pointing in the direction perpendicular to the line of holes, the transmission is almost the same as the one obtained for a single hole [see thin solid line in Fig. 4(a)]. We can visualize this

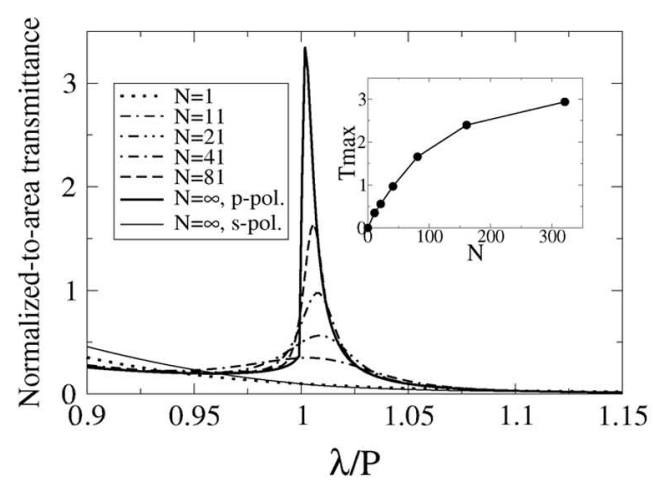

(a)

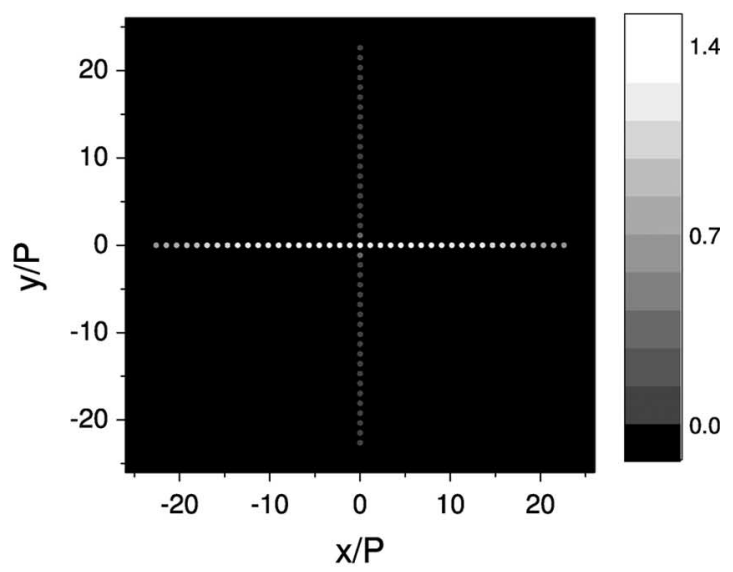

(b)

Fig. 4. (a) Normalized-to-area total transmittance of a linear chain as a function of the wavelength for $a / P=0.25$ and $h / P=0.44$. Different values of the number of holes in the chain $N$ are considered. We assume normal incident and p-polarization. Inbox shows the dependence of the total transmittance $T_{\max }$ as a function of the number of holes. (b) Transmission per hole of a structure composed of two perpendicular lines of holes. The direction of the incident electric field is parallel to the $x$-axis.

property by computing the transmission per hole in a structure composed of two perpendicular chains of holes. Fig. 4(b) shows the corresponding result assuming that the incident electric field is parallel to the $x$-direction. As can be seen in this figure, the transmittance through the holes in the $y$-direction is almost negligible.

\section{Single Hole Flanked by a Periodic Chain of Dimples}

As previously said, within our flexible formalism we can also simulate dimples as well as holes and arbitrary collections formed by dimples and holes. An interesting structure to analyze is a single hole flanked by two finite linear chains of dimples located symmetrically with respect to the central hole (see schematic picture in the top panel of Fig. 5). These linear chains may be placed at the input surface or at the output surface or at both interfaces. In Fig. 5 we show the transmittance (normalized to the incident flux that is impinging at the central hole) through a single hole with $a / P=0.25$, surrounded symmetrically by 80 dimples with the same radii and different depths, $h_{1}$, in a metallic film of thickness $h / P=0.44$. The corrugation is placed at the input surface. As can be seen in 


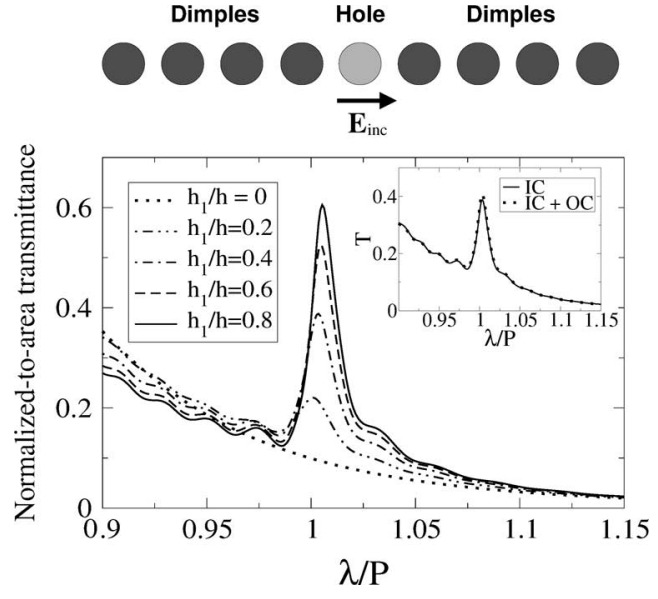

Fig. 5. Schematic picture of the structure under analysis (top panel). Normalized-to-area transmittance through a hole with $a / P=0.25$ and $h / P=$ 0.44 as a function of $\lambda / P$ (main panel). The hole is flanked symmetrically at its input surface by 80 dimples with the same radius and several $h_{1}$ ranging from 0 (no corrugation) to $0.8 h, h$ being the metal thickness. Inbox shows the effect of adding dimples in the output side for $h_{1} / h=0.4$. IC labels the case with dimples only at the input (illuminated) surface of the film while IC+OC corresponds to the case with dimples in both input and output sides.

Fig. 5, again a transmission peak located at around $P$ evolves as $h_{1}$ is increased. The peak value grows almost linearly with $h_{1}$. Interestingly, the total transmission does not increase when two equal additional chains are placed in the output corrugation (see inset of Fig. 5). The transmission spectra for these two cases (corrugation only at the input surface and corrugations at both input and output surfaces) are practically the same for dimples with $h_{1} / h=0.4$.

\section{SUMMARY}

In this paper, we have presented an analysis of the EET appearing in finite size hole arrays. An efficient and accurate theoretical framework that allows us to consider a large number of holes has been presented.

Within this formalism we have studied the total transmission properties of both finite hexagonal hole arrays and linear chains of holes. We have presented circular apertures although we have also worked with squared holes obtaining the same main features in the transmission spectrum. We have shown that we can define a critical size of the structure that determines the number of holes necessary to reproduce the main features corresponding to an infinite hole array. This is interesting from the basic point of view and for possible future applications based on EET effects.

Finally, we have found that a structure formed by a single hole surrounded by two finite linear chains of dimples also presents EET properties and that this result is almost independent of the corrugation in the nonilluminated side.

\section{REFERENCES}

[1] H. A. Bethe, "Theory of diffraction by small holes," Phys. Rev., vol. 66, pp. 163-182, 1944.

[2] C. J. Bouwkamp, "Diffraction theory," Rep. Prog. Phys., vol. XVIII, pp. 35-100, 1954.
[3] A. Roberts, "Electromagnetic theory of diffraction by a circular aperture in a thick, perfectly conducting screen," J. Opt. Soc. Amer. A, Opt. Image Sci., vol. 4, pp. 1970-1983, 1987.

[4] T. W. Ebbesen, H. J. Lezec, H. F. Ghaemi, T. Thio, and P. A. Wolff, "Extraordinary optical transmission through sub-wavelength hole arrays," Nature, vol. 391, pp. 667-669, 1998.

[5] H. F. Ghaemi, T. Thio, D. E. Grupp, T. W. Ebbesen, and H. J. Lezec, "Surface plasmons enhance optical transmission through subwavelength holes," Phys. Rev. B, Condens. Matter, vol. 58, pp. 6779-6782, 1998.

[6] T. Thio, H. F. Ghaemi, H. J. Lezec, P. A. Wolff, and T. W. Ebbesen, "Surface-plasmon-enhanced transmission through hole arrays in cr films," J. Opt. Soc. Amer. B, Opt. Phys., vol. 16, pp. 1743-1748, 1999.

[7] E. Popov, M. Neviere, S. Enoch, and R. Reinisch, "Theory of light transmission through subwavelength periodic hole arrays," Phys. Rev. B, Condens. Matter, vol. 62, pp. 16 100-16 108, 2000.

[8] L. Martín-Moreno, F. J. García-Vidal, H. J. Lezec, K. M. Pellerin, T. Thio, J. B. Pendry, and T. W. Ebbesen, "Theory of extraordinary optical transmission through subwavelength hole arrays," Phys. Rev. Lett., vol. 86, pp. 1114-1117, 2001.

[9] L. Salomon, F. Grillot, A. V. Zayats, and F. de Fornel, "Near-field distribution of optical transmission of periodic subwavelength holes in a metal film," Phys. Rev. Lett., vol. 86, pp. 1110-1113, 2001.

[10] S. A. Darmanyan and A. V. Zayats, "Light tunneling via resonant surface plasmon polariton states and the enhanced transmission of periodically nanostructured metal films: An analytical study," Phys. Rev. B, Condens. Matter, vol. 67, p. 35424, 2003.

[11] C. Genet, M. P. van Exter, and J. P. Woerdman, "Fano-type interpretation of red shifts and red tails in hole array transmission spectra," Opt. Commun., vol. 225, pp. 331-336, 2003.

[12] A. Krishnan, T. Thio, T. J. Kim, H. J. Lezec, T. W. Ebbesen, P. A. Wolff, J. Pendry, L. Martín-Moreno, and F. J. García-Vidal, "Evanescently coupled resonance in surface plasmon enhanced transmission," Opt. Commun., vol. 200, pp. 1-7, 2001.

[13] W. L. Barnes, W. A. Murray, J. Dintinger, E. Devaux, and T. W. Ebbesen, "Surface plasmon polaritons and their role in the enhanced transmission of light through periodic arrays of subwavelength holes in a metal film," Phys. Rev. Lett., vol. 92, p. 107401, 2004.

[14] J. Gómez-Rivas, C. Schotsch, P. H. Bolivar, and H. Kurz, "Enhanced transmission of thz radiation through subwavelength holes," Phys. Rev. B., Condens. Matter, vol. 68, p. 201306, 2003.

[15] M. Beruete, M. Sorolla, I. Campillo, J. S. Dolado, L. Martín-Moreno, J. Bravo-Abad, and F. J. García-Vidal, "Enhanced millimeter-wave transmission through subwavelength hole arrays," Opt. Lett., vol. 29, pp. 25002502, 2004.

[16] — - "Enhanced millimeter wave transmission through quasioptical subwavelength perforated plates," IEEE Trans. Antennas Propag., vol. 53, no. 6, pp. 1897-1903, Jun. 2005.

[17] D. X. Qu and D. Grischkowsky, "Observation of a new type of thz resonance of surface plasmons propagating on metal-film hole arrays," Phys. Rev. Lett., vol. 93, p. 196804, 2004.

[18] E. Moreno, F. J. García-Vidal, and L. Martín-Moreno, "Enhanced transmission and beaming of light via photonic crystal surface modes," Phys. Rev. B, Condens. Matter, vol. 69, p. 121402(R), 2004.

[19] P. Kramper, M. Agio, C. M. Soukoulis, A. Birner, F. Muller R. B. Wehrspohn, U. Gosele, and V. Sandoghdar, "Highly directional emission from photonic crystal waveguides of subwavelength width," Phys. Rev. Lett., vol. 92, p. 113903, 2004.

[20] I. Bulu, H. Caglayan, and E. Ozbay, "Beaming of light and enhanced transmission via surface modes of photonic crystals," Opt. Lett., vol. 30, pp. 3078-3080, 2005.

[21] E. Moreno, A. I. Fernández-Domíngez, J. I. Cirac, F. J. García-Vidal, and L. Martín-Moreno, "Resonant transmission of cold atoms through subwavelength apertures," Phys. Rev. Lett, vol. 95, p. 170406, 2005.

[22] J. Pendry, L. Martín-Moreno, and F. J. García-Vidal, "Mimicking surface plasmons with structured surfaces," Science, vol. 305, pp. 847-848, 2004.

[23] F. J. García-Vidal, L. Martín-Moreno, and J. B. Pendry, "Surfaces with holes in them: New plasmonic metamaterials," J. Opt. A, vol. 7, pp. 97$101,2005$.

[24] J. Bravo-Abad, A. Degiron, F. Przybilla, C. Genet, F. J. García-Vidal, L. Martín-Moreno, and T. W. Ebbesen, "How light emerges from an illuminated array of subwavelength holes," Nature Phys., vol. 2, pp. 120-123, 2006.

[25] H. J. Lezec, A. Degiron, E. Devaux, R. A. Linke, L. Martín-Moreno, F. J. García-Vidal, and T. W. Ebbesen, "Beaming light from a subwavelength aperture," Science, vol. 297, pp. 820-822, 2002. 
[26] L. Martín-Moreno, F. J. García-Vidal, H. J. Lezec, A. Degiron, and T. W. Ebbesen, "Theory of highly directional emission from a single subwavelength aperture surrounded by surface corrugations," Phys. Rev. Lett., vol. 90, p. 167401, 2003.

[27] F. J. García-Vidal, H. J. Lezec, T. W. Ebbesen, and L. Martín-Moreno, "Multiple paths to enhance optical transmission through a single subwavelength slit," Phys. Rev. Lett., vol. 90, p. 213901, 2003.

[28] J. Bravo-Abad, F. J. García-Vidal, and L. Martín-Moreno, "Wavelength de-multiplexing properties of a single aperture flanked by periodic arrays of indentations," Photon. Nanostruct., vol. 1, pp. 55-62, 2003.

[29] F. J. García-Vidal, L. Martín-Moreno, H. J. Lezec, and T. W. Ebbesen, "Focusing light with a single subwavelength aperture flanked by surface corrugations," Appl. Phys. Lett., vol. 83, pp. 4500-4502, 2003.

[30] J. Bravo-Abad, L. Martín-Moreno, and F. J. García-Vidal, "Transmission properties of a single metallic slit: From the subwavelength regime to the geometrical-optics limit," Phys. Rev. E, Stat. Phys. Plasmas Fluids Relat. Interdiscip. Top., vol. 69, p. 26601, 2004.

[31] S. S. Akarca-Biyikli, I. Bulu, and E. Ozbay, "Enhanced transmission of microwave radiation in one-dimensional metallic gratings with subwavelength aperture," Appl. Phys. Lett., vol. 85, pp. 1098-1100, 2004.

[32] W. Srituravanich, N. Fang, C. Sun, Q. Luo, and X. Zhang, "Plasmonic nanolithography," Nano Lett., vol. 4, pp. 1085-1088, 2004.

[33] F. Miyamaru and M. Hangyo, "Finite size effect of transmission property for metal hole arrays in subterahertz region," Appl. Phys. Lett., vol. 84, pp. 2742-2744, 2004

[34] J. Bravo-Abad, F. J. García-Vidal, and L. Martín-Moreno, "Resonant transmission of light through finite chains of subwavelength holes in a metallic film.," Phys. Rev. Lett., vol. 93, p. 227401, 2004.

[35] L. Martín-Moreno and F. J. García-Vidal, "Optical transmission through circular hole arrays in optically thick metal films," Opt. Express, vol. 12, pp. 3619-3628, 2004

[36] R. Gordon and A. G. Brolo, "Increased cut-off wavelength for a subwavelength hole in a real metal," Opt. Express, vol. 13, pp. 1933-1938, 2005.

[37] P. M. Morse and H. Feshbach, Methods of Theoretical Physics. New York: McGraw-Hill, 1953.
[38] P. M. Bell, J. B. Pendry, L. Martín-Moreno, and A. J. Ward, "A program for calculating photonic band structures and transmission coefficients of complex structures," Comput. Phys. Commun., vol. 85, pp. 306-322, 1995.

[39] E. Moreno, D. Erni, and C. Hafner, "Band structure computations of metallic photonic crystals with the multiple multipole method," Phys. Rev. B, Condens. Matter, vol. 65, p. 155120, 2002.

Jorge Bravo-Abad received the M.S. degree in physics from Universidad Autónoma de Madrid, Spain, in 2001, where he is currently working toward the Ph.D. degree in the Departamento de Fsica Teórica de la Materia Condensada.

Luis Martín-Moreno received the M.S. and Ph.D. degrees in physics from Universidad Autónoma de Madrid, Madrid, Spain, in 1985 and 1989, respectively.

Since 1994, he has been an Associate Professor at the Universidad de Zaragoza, Zaragoza, Spain.

Francisco J. García-Vidal received the M.S. and Ph.D. degrees in physics from Universidad Autónoma de Madrid, Madrid, Spain, in 1988 and 1992, respectively.

Since 1996, he has been an Associate Professor at the same university. 